\title{
Protein-repellent and antibacterial nanocomposite for Class- $V$ restorations to inhibit periodontitis-related pathogens
}

\author{
Lin Wang ${ }^{1,2}$, Xianju Xie ${ }^{2,3}$, Satoshi Imazato ${ }^{4}$, \\ Michael D. Weir ${ }^{2}$, Mark A. Reynolds ${ }^{2}$, Hockin H. K. Xu ${ }^{2,5,6}$
}

${ }^{1}$ VIP Integrated Department, School and Hospital of Stomatology, Jilin University, Changchun, 130011, China

${ }^{2}$ Department of Endodontics, Periodontics and Prosthodontics, University of Maryland School of Dentistry, Baltimore, MD 21201, USA

${ }^{3}$ Department of Orthodontics, School of Stomatology, Capital Medical University, Beijing, China

${ }^{4}$ Department of Biomaterials Science, Osaka University Graduate School of Dentistry, Osaka, Japan

${ }^{5}$ Center for Stem Cell Biology \& Regenerative Medicine, University of Maryland School of Medicine, Baltimore, MD 21201, USA

${ }^{6}$ Department of Mechanical Engineering, University of Maryland Baltimore County, Baltimore County, MD 21250, USA

For: Materials Science and Engineering: C (Materials for Biological Applications) (Submitted in March 2016, revised and resubmitted in April 2016)

\section{Correspondence:}

Dr. Hockin $\mathrm{Xu}$, Department of Endodontics, Periodontics and Prosthodontics, University of Maryland Dental School, Baltimore, MD 21201, email: hxu@umaryland.edu. Dr. Lin Wang, VIP Integrated Department, Stomatological Hospital of Jilin University, Changchun, China, email: wanglin1982@jlu.edu.cn.

Short title: Multifunctional nanocomposite to inhibit periodontal pathogens

Key words: Dental composite, Class V restoration, antibacterial, protein repellent, calcium phosphate nanoparticle, periodontal pathogens 


\section{Abstract}

The objectives of this study were to develop a bioactive dental composite and investigate the effects of 2-methacryloyloxyethyl phosphorylcholine (MPC) and dimethylaminohexadecyl methacrylate (DMAHDM) in Class V composite on mechanical properties, water sorption, protein adsorption, and inhibition of four species of periodontitis-related biofilms for the first time. The resin consisted of ethoxylated bisphenol A dimethacrylate (EBPADMA) and pyromellitic glycerol dimethacrylate (PMGDM). DMAHDM, MPC and nanoparticles of amorphous calcium phosphate (NACP) were incorporated into the resin. Four species $(P$. gingivalis, $P$. intermedia, A. actinomycetemcomitans and $F$. nucleatum) were tested for biofilm colony-forming units (CFU), live/dead, metabolic activity, and polysaccharide production. The results showed that adding DMAHDM and MPC to the composite did not compromise the mechanical properties $(p>0.1)$, with acceptable water sorption values. Composite with $3 \%$ MPC reduced protein adsorption to $1 / 9$ that of a commercial composite $(p<0.05)$. For all four species, the composite with 3\% DMAHDM $+3 \%$ MPC had much greater reduction in biofilms than using DMAHDM or MPC alone $(p<0.05)$. Biofilm CFU was reduced by about 4 orders of magnitude via 3\% DMAHDM +3\% MPC, compared to control. The inhibition efficacy for the four species was: $P$. gingivalis $>P$ intermedia $=A$. actinomycetemcomitans > F. nucleatum. In conclusion, a novel bioactive composite with 3\% DMAHDM and 3\% MPC achieved the greatest reduction in biofilm growth, metabolic activity and polysaccharide of four periodontal pathogens. The new composite is promising for Class V restorations especially with subgingival margins to inhibit periodontal pathogens, combat periodontitis and protect the periodontium. 


\section{Introduction}

The aging of population is associated with major changes in oral disease patterns [1]. Studies have confirmed an increasing incidence of root caries among the elderly, in part attributable to the increasing proportion of elderly people retaining their natural teeth $[2,3]$. Gingival recession and reduced saliva in seniors can contribute to root caries [3], which can be treated with a Class V restoration. Class V restorations with subgingival margins may be difficult to clean and provide pockets for periodontal bacterial growth. This in turn could enhance the development of periodontitis and the loss of the tooth's attachment. It is accepted that microbial biofilms are the principal aetiological factor of periodontitis which eventually leads to tooth loss [4]. However, current resin-based Class V restorations do not inhibit biofilm growth, and instead may even accumulate more biofilms and plaque [5].

Three main bacterial species frequently detected in subgingival plaque of periodontitis and peri-implantitis sites are: Porphyromonas gingivalis (P. gingivalis), Prevotella intermedia (P. intermedia) and Aggregatibacter actinomycetemcomitans (A. actinomycetemcomitans) [6]. They can produce virulence factors in periodontal pockets, leading to progressive loss of the alveolar bone and periapical bone [6]. P. gingivalis may act as a keystone pathogen in periodontitis $[6,7]$. It impairs innate immunity in ways that alter the growth the biofilm, triggering a destructive change in the normally homeostatic host-microbiota interplay in the periodontium [7]. The second species, $P$. intermedia, is associated with pregnancy gingivitis and periodontitis, because this species can use estrogen and progesterone as an essential source of growth instead of using vitamin $\mathrm{K}$ [8]. The third species, A. actinomycetemcomitans is associated with localized aggressive periodontitis [9]. In addition, a fourth species, 
Fusobacterium nucleatum ( $F$. nucleatum) is a Gram-negative anaerobe. The ability of $F$. nucleatum to co-aggregate with many plaque bacteria suggests that it acts as a microbial bridge between early and late colonizers [10]. F. nucleatum is also an initiator organism by promoting physico-chemical changes in the gingival sulcus allowing periodontal pathogenic successors to establish and proliferate [11]. Therefore, these four periodontitis-related pathogens were selected in the present study.

Composites are widely used in dental practice as filling materials due to their esthetics and direct-filling capabilities [12,13]. Previous efforts have led to significant improvements in resin compositions, filler particles and polymerization [14-16]. Composite are challenged with accumulation of more biofilms than other restorative materials [5], which could result in secondary caries. Therefore, efforts were made to develop antibacterial dental resins [17-19]. Quaternary ammonium methacrylates (QAMs) were copolymerized in resins for antibacterial functions [20-22]. Resins with 12-methacryloyloxydodecylpyridinium bromide (MDPB) were effective in contact-killing of bacteria [22]. A quaternary ammonium dimethacrylate (QADM) also achieved strong bacterial effects [23]. The antibacterial activity of QAMs increased when alkyl chain length (CL) was increased from 5 to 16 [24]. Resins containing dimethylaminohexadecyl methacrylate (DMAHDM) with CL of 16 had a strong activity [24].

Salivary protein coating on resin could decrease the efficacy of "contact-inhibition" [21, 25]. Therefore, efforts were made to develop protein-repellent functions [26]. 2-methacryloyloxyethyl phosphorylcholine (MPC) is a methacrylate with phospholipid polar groups and is a common biopolymer [27]. Polymers containing MPC are known to reduce protein adsorption and bacterial adhesion [28, 29]. In our previous studies, MPC-containing 
resins showed strong protein-repellent properties and inhibition against cariogenic bacteria [30, 31]. However, to date, there has been no report on the effects of Class $\mathrm{V}$ composite containing MPC and DMAHDM on biofilms of periodontitis-related pathogens.

Our recent study used a resin consisting of ethoxylated bisphenol A dimethacrylate (EBPADMA) and pyromellitic dianhydride glycerol dimethacrylate (PMGDM) [32]. When filled with nanoparticles of amorphous calcium phosphate (NACP), the composite could be recharged with calcium $(\mathrm{Ca})$ and phosphate $(\mathrm{P})$ ions to have long-term ion release [32]. The objectives of the present study were to incorporate MPC and DMAHDM into the rechargeable NACP nanocomposite for Class V restorations, and to investigate the inhibition of biofilms of four periodontal pathogens $(P$ gingivalis, $P$. intermedia, $A$. actinomycetemcomitans, and $F$. nucleatum) for the first time. It was hypothesized that: (1) Incorporating MPC and DMAHDM into the rechargeable NACP composite would not compromise the mechanical properties; (2) MPC and DMAHDM together would reduce biofilms of the four periodontal pathogens much more than MPC or DMAHDM alone; (3) Different periodontal pathogens would have different biofilm properties when growing on the rechargeable NACP composite containing DMAHDM and MPC.

\section{Materials and Methods}

\subsection{Preparation of composites containing DMAHDM and MPC}

EBPADMA (Sigma-Aldrich, St. Louis, MO, USA) and PMGDM (Esstech, Essington, PA, USA) were mixed at an EBPADMA:PMGDM mass ratio of 1:1, which was render light-curable with $0.2 \%$ camphorquinone and $0.8 \%$ ethyl 4-N,N-dimethylaminobenzoate [32]. 
This resin is referred to as EBPM.

MPC was obtained commercially (Sigma-Aldrich) which was synthesized via a method reported previously [27]. MPC powder was incorporated into the EBPM resin at $\mathrm{MPC} /(\mathrm{EBPM}+\mathrm{MPC})$ mass fractions of $0 \%, 5 \%, 10 \%$, and $15 \%$. MPC mass fractions greater than $15 \%$ were not included due to mechanical property loss in preliminary studies.

DMAHDM was synthesized using a modified Menschutkin reaction in which a tertiary amine group was reacted with an organohalide [33]. Briefly, $10 \mathrm{mmol}$ of 2-(dimethylamino)ethyl methacrylate (DMAEMA, Sigma-Aldrich) and $10 \mathrm{mmol}$ of 1-bromohexadecane (BHD, TCI America, Portland, OR, USA) were combined with $3 \mathrm{~g}$ of ethanol in a $20 \mathrm{~mL}$ scintillation vial. The vial was stirred at $70{ }^{\circ} \mathrm{C}$ for $24 \mathrm{~h}$. The solvent was then removed via evaporation, yielding DMAHDM as a clear, colorless, and viscous liquid [23]. DMAHDM was mixed with either the EBPM resin or the EBPM-MPC resin at a DMAHDM mass fraction of $10 \%$. The $10 \%$ mass fraction followed a previous study [23].

In addition, $20 \%$ by mass of NACP fillers were incorporated into the resin [32]. The reason for including NACP was for $\mathrm{Ca}$ and $\mathrm{P}$ ion release which could remineralize tooth lesions as shown previously [34]. NACP were synthesized using a spray-drying technique as previously described [35]. Briefly, calcium carbonate and dicalcium phosphate anhydrous were dissolved in acetic acid to produce $\mathrm{Ca}$ and $\mathrm{P}$ concentrations of $8 \mathrm{mmol} / \mathrm{L}$ and $5.333 \mathrm{mmol} / \mathrm{L}$, respectively, thus yielding a $\mathrm{Ca} / \mathrm{P}$ molar ratio of 1.5 , the same as that for $\mathrm{ACP}$ $\left[\mathrm{Ca}_{3}\left(\mathrm{PO}_{4}\right)_{2}\right]$. This solution was sprayed into a heated chamber of the spray-drying machine. An electrostatic precipitator was used to collect the dried particles. This produced NACP with a mean particle size of $116 \mathrm{~nm}$ [35]. Barium boroaluminosilicate glass particles with a 
median size of $1.4 \mu \mathrm{m}$ were used as a co-filler (Caulk/Dentsply, Milford, DE, USA), which was silanized with 4\% 3-methacryloxypropyltrimethoxysilane and 2\% n-propylamine [35]. The NACP and glass filler mass fractions were $20 \%$ and $50 \%$, respectively, with a total filler level of $70 \%$, yielding a cohesive composite paste. Since the resin mass fraction in the composite was $30 \%$, the DMAHDM mass fraction in the final composite was $3 \%$. The MPC mass fractions in the composite were $0 \%, 1.5 \%, 3 \%$, and $4.5 \%$, respectively.

Eight composites were thus fabricated, using a 4x2 full-factorial design with 4 levels of MPC $(0 \%, 1.5 \%, 3 \%, 4.5 \%)$ and 2 levels of DMAHDM $(0 \%, 3 \%)$ :

(1) $20 \% \mathrm{NACP}+50 \%$ glass $+30 \%$ EBPM (referred to as EBPM composite control);

(2) $20 \% \mathrm{NACP}+50 \%$ glass $+27 \% \mathrm{EBPM}+1.5 \% \mathrm{MPC}(\mathrm{EBPM}+1.5 \mathrm{MPC})$;

(3) $20 \% \mathrm{NACP}+50 \%$ glass $+27 \% \mathrm{EBPM}+3 \% \mathrm{MPC}(\mathrm{EBPM}+3 \mathrm{MPC})$

(4) $20 \% \mathrm{NACP}+50 \%$ glass $+27 \% \mathrm{EBPM}+4.5 \% \mathrm{MPC}(\mathrm{EBPM}+4.5 \mathrm{MPC} ")$

(5) $20 \% \mathrm{NACP}+50 \%$ glass + 27\% EBPM + 3\% DMAHDM (EBPM+3DMAHDM);

(6) $20 \% \mathrm{NACP}+50 \%$ glass $+25.5 \%$ EBPM $+3 \%$ DMAHDM $+1.5 \% \mathrm{MPC}$ (EBPM+3DMAHDM+1.5 MPC);

(7) $20 \% \mathrm{NACP}+50 \%$ glass $+24 \% \mathrm{EBPM}+3 \% \mathrm{DMAHDM}+3 \% \mathrm{MPC}$ (EBPM+3DMAHDM+3MPC);

(8) $20 \% \mathrm{NACP}+50 \%$ glass $+22.5 \%$ EBPM $+3 \%$ DMAHDM $+4.5 \% \mathrm{MPC}$ (EBPM+3DMAHDM+4.5MPC).

In addition, a commercial composite control was also tested (Heliomolar, Ivoclar, Ontario, Canada). Heliomolar contained silica and ytterbium-trifluoride fillers with particle sizes of $40-200 \mathrm{~nm}$ at a filler level of $66.7 \%$. Heliomolar was selected because it is indicated 
for Class I and II restorations as well as Classes V restorations for root caries.

\subsection{Mechanical properties}

Each composite paste was placed into a mold of $2 \times 2 \times 25 \mathrm{~mm}$ and photo-cured (Triad 2000, Dentsply, York, PA, USA) for 1 min on each open side of the specimen. The irradiance of the Triad 2000 was previously measured at the specimen position to be $65 \mathrm{~mW} / \mathrm{cm}^{2}$ [36]. Six specimens per composite were made. The specimens were stored at $37{ }^{\circ} \mathrm{C}$ in water for 24 h. Flexural strength and elastic modulus were measured using a three-point flexural test with a $10 \mathrm{~mm}$ span at a crosshead-speed of $1 \mathrm{~mm} / \mathrm{min}$ on a computer-controlled Universal Testing Machine (5500R, MTS, Cary, NC) [35]. Flexural strength was calculated by: $\mathrm{S}=3 \mathrm{P}_{\max } \mathrm{L} /$ $\left(2 b^{2}\right)$, where $P_{\max }$ is the fracture load, $\mathrm{L}$ is span, $\mathrm{b}$ is specimen width and $\mathrm{h}$ is thickness. Elastic modulus was calculated by: $\mathrm{E}=(\mathrm{P} / \mathrm{d})\left(\mathrm{L}^{3} /\left[4 \mathrm{bh}^{3}\right]\right)$, where load $\mathrm{P}$ divided by displacement $d$ is the slope in the linear elastic region [35].

\subsection{Water sorption measurement}

Five specimens per composite were made for water sorption $\left(\mathrm{W}_{\mathrm{SP}}\right)$ assessment following ISO 4049:2009 [37]. Each composite paste was placed into a mold with $15 \mathrm{~mm}$ in diameter and $1 \mathrm{~mm}$ in thickness. The specimen was light-cured (Triad 2000) for $1 \mathrm{~min}$ on each open side of the mold and then incubated at $37{ }^{\circ} \mathrm{C}$ for $24 \mathrm{~h}$. The specimens were then dried in a vacuum desiccator at $37{ }^{\circ} \mathrm{C}$ for $24 \mathrm{~h}$ until a constant mass was reached $\left(\mathrm{M}_{1}\right)$. The diameter and height of each specimen were measured individually to calculate the volume V. Then the specimens were immersed in distilled water at $37{ }^{\circ} \mathrm{C}$ for 7 days (d). After immersion, the specimen surfaces were gently wiped absorbent paper and the specimen was weighed to 
obtain $\mathrm{M}_{2}$. The specimen was returned to the desiccator under vacuum for $7 \mathrm{~d}$, and then weighed to obtain $\mathrm{M}_{3}$. $\mathrm{W}_{\mathrm{SP}}$ was calculated as: $\mathrm{W}_{\mathrm{SP}}=\left(\mathrm{M}_{2}-\mathrm{M}_{3}\right) / \mathrm{V}$ [37].

\subsection{Measurement of protein adsorption}

The composite with 3\% DMAHDM plus 4.5\% MPC (EBPM+3DMAHDM+4.5MPC) had a higher water sorption than ISO requirement; therefore, it was not included in the subsequent protein-repellent and biofilm tests. In addition, preliminary study showed that $1.5 \%$ MPC was not as effective as $3 \%$ MPC to repel proteins. Hence, the following five composites were tested in protein and biofilm experiments: Heliomolar, EBPM composite control, EBPM+3MPC, EBPM+3DMAHDM, and EBPM+3DMAHDM+3MPC.

Each composite paste was filled into disk molds of $9 \mathrm{~mm}$ in diameter and $2 \mathrm{~mm}$ in thickness. Five disks were made for each composite. They were photo-cured with 1 min on each open side (Triad 2000) and immersed in water at $37^{\circ} \mathrm{C}$ for $24 \mathrm{~h}$. Protein adsorption was determined using a micro bicinchoninic acid (BCA) method [29, 38]. Each disk was immersed in phosphate buffered saline (PBS) for $2 \mathrm{~h}$, and then immersed in $4.5 \mathrm{~g} / \mathrm{L}$ bovine serum albumin (BSA, Sigma-Aldrich) solution at $37{ }^{\circ} \mathrm{C}$ for $2 \mathrm{~h}[29,38]$. The disks were rinsed with PBS, then immersed in $1 \%$ sodium dodecylsulfate (SDS) in PBS and sonicated for 20 min to detach the BSA adsorbed on the disk [29, 38]. A protein analysis kit (micro BCA, Fisher Scientific, Pittsburgh, PA, USA) was used to determine BSA concentration in the SDS solution [30, 31]. $25 \mu \mathrm{L}$ of SDS solution and $200 \mu \mathrm{L}$ of BCA reagent were mixed into the wells of a 96-well plate and incubated at $60{ }^{\circ} \mathrm{C}$ for $30 \mathrm{~min}$. The absorbance at $562 \mathrm{~nm}$ was measured via a microplate reader (SpectraMax M5, Molecular Devices, Sunnyvale, CA, 
USA). Standard curves were prepared using the BSA standard [29, 38].

\subsection{Bacteria culture}

The use of bacteria was approved by University of Maryland Institutional Review Board. P. gingivalis ATCC33277, P. intermedia ATCC 25611, A. actinomycetemcomitans ATCC 43717, and F. nucleatum ATCC 25586 were all obtained from ATCC (Manassas, VA, USA). Each species was grown in a tryptic soy broth (TSB, Sigma) supplemented with yeast extract (5 g/L), L-cysteine hydrochloride $(0.5 \mathrm{~g} / \mathrm{L})$, hemin $(5 \mathrm{mg} / \mathrm{L})$ and menadione $(1 \mathrm{mg} / \mathrm{L})$ at $37^{\circ} \mathrm{C}$ anaerobically $\left(90 \% \quad \mathrm{~N}_{2}, 5 \% \quad \mathrm{CO}_{2}, 5 \% \mathrm{H}_{2}\right)$ [8]. The inoculum was adjusted to $10^{7}$ colony-forming unit counts $(\mathrm{CFU} / \mathrm{mL})$, based on the standard curve of $\mathrm{OD}_{600 \mathrm{~nm}}$ versus $\mathrm{CFU} / \mathrm{mL}$ for each species [8].

\subsection{Biofilm formation on composite}

Photo-cured composite disks were immersed in $200 \mathrm{~mL}$ of water and magnetically-stirred with a bar at $100 \mathrm{rpm}$ for $1 \mathrm{~h}$ to remove any uncured monomers [39]. The disks were then sterilized with ethylene oxide (AnproleneAN 74i, Andersen, Haw River, NC, USA) and de-gassed for 7 days.

Saliva collection was approved by the University of Maryland Baltimore Institutional Review Board (HP-00050407). Saliva was collected from fifteen healthy adult donors having natural dentition without active caries or periopathology, and without the use of antibiotics within the last 3 months. The donors did not brush teeth for 24 hours and abstained from food and drink intake for 2 hours prior to donating saliva. An equal volume of saliva from each of 
the fifteen donors was combined to form the saliva sample. Saliva was centrifuged at 3000 rpm for 20 min to remove cellular debris. The supernatant was filter-sterilized through sterile $0.22 \mu \mathrm{m}$ filters (VWR International, Radnor, PA, USA). Sterile saliva was used to coat a salivary pellicle on composite disk by immersing the disk in saliva for $2 \mathrm{~h}$ at $37^{\circ} \mathrm{C}$ [40].

Each bacteria species was used individually to form single-species biofilms following a previous study [8]. The salivary pellicle-coated composite disks were transferred to a new 24-well plate, and each bacterial species was inoculated at a concentration of $10^{7} \mathrm{CFU} / \mathrm{mL}$ in $1.5 \mathrm{~mL}$ medium in each well. After $24 \mathrm{~h}$, the disks with adherent biofilms were transferred to new 24-well plates filled with fresh medium and incubated for $24 \mathrm{~h}$. This totaled 2 days of culture which was suitable to form biofilm, following previous studies [8, 41].

\subsection{Live/dead bacteria imaging}

Each disk with 2-day biofilms was washed with cysteine peptone water (CPW) to remove the non-adherent bacteria. Live/dead bacterial kit (Molecular Probes, Eugene, OR, USA) was used following the manufacturer's instructions. A mixture of $2.5 \mu \mathrm{M}$ SYTO 9 and $2.5 \mu \mathrm{M}$ propidium iodide was used to stain each sample for $15 \mathrm{~min}$. Live bacteria were stained with SYTO 9 to emit a green fluorescence. Bacteria with compromised membranes were stained with propidium iodide to emit a red fluorescence. Biofilms were imaged with an inverted epifluorescence microscope (TE2000-S, Nikon, Melville, NY, USA). Three disks were tested for each of the five composites with each bacterial species, using a total of 60 disks for live/dead staining. Five random images were taken for each disk, yielding 15 images for each composite with each bacterial species. 


\subsection{CFU counts}

Twenty-four disks were made for each composite, with six disks for each bacterial species. Biofilms were formed by culturing for 2 days as described above. Disks were transferred into vials with $2 \mathrm{~mL} \mathrm{CPW}$, and the biofilms were harvested by scraping and sonication/vortexing (Fisher, Pittsburg, PA, USA). Tryptic soy blood agar plates (supplemented with $5 \mathrm{~g} / \mathrm{L}$ yeast extract, $0.5 \mathrm{~g} / \mathrm{L}$ L-cysteine hydrochloride, $5 \mathrm{mg} / \mathrm{L}$ hemin, 1 $\mathrm{mg} / \mathrm{L}$ menadione, 5\% sheep blood) were used, following ATCC instructions. Biofilm suspensions were serially diluted, spread onto agar plates and incubated at $37{ }^{\circ} \mathrm{C}$ anaerobically for $48 \mathrm{~h}$. Then, the number of colonies was counted by a colony counter (Reichert, NY, USA), which was used with the dilution factor to calculate CFU counts [24].

\subsection{MTT metabolic assay}

Twenty-four disks were made for each composite for the metabolic assay, with six disks for each bacterial species. The MTT assay is a colorimetric assay that relies on the enzymatic reduction of a yellow tetrazolium salt, 3-(4, 5-dimethylthiazol-2-yl)-2, 5-diphenyltetrazolium bromide (MTT), which forms a purple formazan crystal in metabolically active cells [42]. Disks with 2-day biofilms were transferred into new 24-well plates with $1 \mathrm{~mL}$ of MTT dye $\left(0.5 \mathrm{mg} / \mathrm{mL}\right.$ MTT in PBS) in each well and incubated at $37{ }^{\circ} \mathrm{C}$ in $5 \% \mathrm{CO}_{2}$ for $1 \mathrm{~h}$. The disks were then transferred to new 24-well plates, and $1 \mathrm{~mL}$ of dimethyl sulfoxide (DMSO) was added to each well to solubilize the formazan crystals [42]. The plates were incubated for 20 min with gentle mixing at room temperature in the dark. After mixing via pipetting, 200 
$\mu \mathrm{L}$ of the DMSO solution was transferred to a 96 -well plate, and the absorbance at $\mathrm{OD}_{540 \mathrm{~nm}}$ was measured via the microplate reader (SpectraMax M5). A higher absorbance is related to a higher metabolic activity in the biofilm on the disks [42].

\subsection{Polysaccharide production measurement}

Twenty-four disks were made for each composite for the polysaccharide measurement, with six disks for each bacterial species. The water-insoluble polysaccharide in the extracellular polymeric substance (EPS) of biofilms was determined using a phenol-sulfuric acid method [8]. Each disk with 2-day biofilm was immersed in a vial with 2 $\mathrm{mL}$ CPW, and the biofilm was collected by sonication/votexing. Centrifugation yielded a precipitate, which was rinsed with PBS and resuspended in $1 \mathrm{~mL}$ of de-ionized water. Then, 1 $\mathrm{mL}$ of $6 \%$ phenol solution were added to the vial, followed by $5 \mathrm{~mL}$ of $95-97 \%$ sulfuric acid [8]. The vial was incubated for $30 \mathrm{~min}$; then, $100 \mu \mathrm{L}$ was transferred to a 96 -well plate. The amount of polysaccharide was determined by measuring the absorbance at $\mathrm{OD}_{490 \mathrm{~nm}}$ with the microplate reader. Five glucose concentrations of 0, 5, 10, 20, 50 and $100 \mathrm{mg} / \mathrm{mL}$ were used as standard in the conversion of OD readings to polysaccharide concentrations [8].

\subsection{Statistical analysis}

All data were checked for normal distribution with the Kolmogorov-Smirnov test. Significant differences were tested by one-way and two-way analyses of variance (ANOVA). Tukey's post-hoc test was used for pairwise comparisons. Statistical analyses were performed by SPSS 19.0 software (SPSS, Chicago, IL, USA) at an alpha of 0.05. 


\section{Results}

The mechanical properties of composites are plotted in Fig. 1: (A) Flexural strength, (B) elastic modulus (mean $\pm \mathrm{SD} ; \mathrm{n}=6$ ), and $(\mathrm{C})$ water sorption (mean $\pm \mathrm{SD} ; \mathrm{n}=5$ ). Only the composite with 3\% DMAHDM + 4.5\% MPC had significantly lower strength and elastic modulus $(p<0.05)$; all other composites had similar strengths and elastic moduli $(p>0.1)$. These results showed that adding up to 3\% MPC and 3\% DMAHDM did not compromise the strength and elastic modulus, compared to control $(p>0.1)$. The composites had significantly different water sorption values $(\mathrm{p}<0.05)$.

Protein adsorption on composites is plotted in Fig. 2 (mean $\pm S D ; n=6)$. Adding 3\% DMAHDM in composite had no effect on protein adsorption $(p>0.1)$. Adding $3 \%$ MPC in composite substantially decreased the protein adsorption by about an order of magnitude, compared to that without MPC and the commercial control $(p<0.05)$.

Representative live/dead images of 2-day biofilms for the $5 \times 4$ full-factorial design are shown in Fig. 3. The five composites (commercial control, EBPM composite control, EBPM+3MPC, EBPM+3DMAHDM, and EBPM+3DMAHDM+3MPC) are labeled on the left side. The four species ( $P$. gingivalis, $P$. intermedia, A. actinomycetemcomitans, and $F$. nucleatum) is labeled on the top. Live bacteria were stained green, and bacteria with compromised membranes were stained red. For all four species, the two control composites were covered by live bacteria. In contrast, composite with $3 \%$ MPC had much less bacterial adhesion. Composite with 3\% DMAHDM had substantial dead bacteria. Composite with 3\% DMAHDM + 3\% MPC had much less bacterial adhesion, and the bacteria were mostly dead. 
The CFU counts of 2-day biofilms on composites are plotted in Fig. 4 for: (A) $P$. gingivalis, (B) P. intermedia, (C) A. actinomycetemcomitans, and (D) F. nucleatum (mean \pm $\mathrm{SD} ; \mathrm{n}=6$ ). Different species had different CFU on the control composites, some having close to $10^{10} \mathrm{CFU} /$ disk, and some with less than $10^{9} \mathrm{CFU} /$ disk. For each species, the commercial composite and experimental composite control had similar CFU $(p>0.1)$. Incorporating DMAHDM or MPC alone into the composite decreased the CFU $(p<0.05)$. The composite with double agents, 3\% DMAHDM $+3 \%$ MPC, reduced the CFU of different species differently, some by slightly less than $4 \log$, others by more than $4 \log$. The CFU of $P$. gingivalis, $P$ intermedia, A. actinomycetemcomitans and $F$. nucleatum biofilms on EBPM+3DMAHDM+3MPC were reduced to $0.008 \%, 0.009 \%, 0.009 \%$ and $0.014 \%$, respectively, of the corresponding CFU on control composite.

The metabolic activity data of 2-day biofilms are plotted in Fig. 5 for: (A) P. gingivalis, (B) P. intermedia, (C) A. actinomycetemcomitans, and (D) F. nucleatum (mean $\pm \mathrm{SD} ; \mathrm{n}=6$ ). For each species, the commercial composite and the EBPM composite control had similar values ( $p>0.1)$. Compared to controls, incorporation of DMAHDM or MPC alone greatly reduced the metabolic activity of biofilms. EBPM+3DMAHDM+3MPC had the least metabolic activity of biofilms for all four periodontal pathogens $(p<0.05)$.

The results of polysaccharide production by biofilms on composites are plotted in Fig. 6 for: (A) P. gingivalis, (B) P. intermedia, (C) A. actinomycetemcomitans, and (D) F. nucleatum (mean $\pm \mathrm{SD} ; \mathrm{n}=6$ ). The commercial composite and EBPM composite control had similar polysaccharide amounts $(p>0.1)$. Polysaccharide on EBPM+3DMAHDM+3MPC was much less than that on controls $(p<0.05)$. These results showed that EBPM+3DMAHDM+3MPC 
had potent inhibition on periodontal pathogens and their synthesis of the extracellular matrix.

\section{Discussion}

The present study developed a nanocomposite for Class $\mathrm{V}$ restorations containing MPC and DMAHDM to inhibit periodontitis-related pathogens which is especially beneficial in root caries restorations with subgingival margins. The hypotheses were proven that adding MPC and DMAHDM in composite did not decrease the mechanical properties; MPC + DMAHDM had an anti-biofilm potency against periodontal pathogens much greater than MPC or DMAHDM alone; and different periodontal pathogens exhibited different biofilm properties and killing efficacy via DMAHDM and MPC. The composite with 3\% DMAHDM $+3 \% \mathrm{MPC}$ is promising for Class $\mathrm{V}$ restorations to inhibit periodontal biofilms, reducing CFU by nearly $4 \log$ for all four periodontitis-related pathogens.

Mechanical properties are important for tooth restorations. Photo-polymerization affects the mechanical properties of composites. Composite restorations are usually cured with narrow-band light-emitting diode (LED) units in clinics. In the present study, the composites were cured using a Triad 2000 (broadband tungsten halogen) unit with a lower intensity than LED curing units. Our previous study showed that curing with the Triad 2000 led to a sufficient degree of polymerization conversion (DC) of $87.3 \%$ [43]. Another study used the Triad 2000 curing unit and reached a DC of 89\% [44]. These good DC values are consistent with a report showing that three composites exhibited similar mechanical properties when cured via different light-cure units with LED versus halogen bulbs [45]. 
Another factor that affects mechanical properties is water-sorption by the composite. Several factors influence water-sorption, including the hydrophilicity of the polymer matrix, crosslinking density, and filler level [46]. MPC is hydrophilic, and the water-sorption of composite increased with increasing MPC content in the composite. Indeed, the water-sorption of EBPM+3DMAHDM+4.5MPC was $47 \mu \mathrm{g} / \mathrm{mm}^{3}$, which exceeded the ISO requirement of $\leq 40 \mu \mathrm{g} / \mathrm{mm}^{3}$ [37]. While a commercial resin-modified glass ionomer (with uses including Class V restorations) had a water-sorption of $140 \mu \mathrm{g} / \mathrm{mm}^{3}$ [47], to follow the ISO suggestion the EBPM+3DMAHDM+4.5MPC was excluded from biofilm experiments in the present study.

Periodontal disease is prevalent and a significant public health problem [48]. A survey showed that periodontal disease in the U.S. affects nearly half (45.9\%) of the population 30 years of age and older [48]. The increasing prevalence of root caries is associated with the increasing prevalence of periodontal disease [49]. Class V restorations with subgingival margins can be difficult to clean, with pockets for periodontal pathogen growth. This could set off a vicious cycle, leading to more gingival recession, which in turn leads to more root exposure and root caries. Therefore, it would be highly desirable to develop a bioactive composite for Class $\mathrm{V}$ restorations with antibacterial function against periodontal pathogens to inhibit local periodontitis and protect the periodontal attachment.

In the present study, $P$. gingivalis, $P$. intermedia, A. actinomycetemcomitans and $F$. nucleatum were selected to investigate the inhibition of periodontal pathogens, for the following reasons. First, $P$. gingivalis, $P$. intermedia and A. actinomycetemcomitans are frequently detected in gingivitis [50]. Gingivitis does not affect the underlying supporting 
structures of the teeth and is reversible. However, when gingivitis is left untreated, it can advance to periodontitis in which the inner layer of the gum and bone would pull away from the teeth to form pockets, followed by bone resorption and eventually leading to tooth loss [51]. Second, P. gingivalis, $P$. intermedia and A. actinomycetemcomitans are detected in subgingival plaque of periodontitis and peri-implantitis sites [51]. They produce virulence factors in periodontal pockets, leading to loss of the alveolar bone and periapical bone [51]. Third, F. nucleatum is not only detected in advanced periodontitis, but also plays potential synergistic effects with $P$. gingivalis on virulence [10]. Unfortunately, current dental composites for Class V restorations had no antibacterial function [5].

Glass ionomer materials are used in Class V restorations. Glass ionomer materials are widely used due to their fluoride release, good biocompatibility with dental pulp tissues, and bonding to tooth structures. Fluoride release may have antimicrobial and remineralization effects. However, previous studies indicated that a commercial resin-modified glass ionomer was not potent enough to inhibit bacterial growth and biofilm formation [30,31]. In addition, for anterior teeth, cervical lesions need to be esthetic, which can be better satisfied with a composite. Therefore, the present study focused on composites for Class V restorations.

In developing a new Class V composite to inhibit periodontal pathogens, our approach is based on the fact that saliva-derived protein adsorption on material surface is a prerequisite in bacteria attachment [52]. Therefore, making a protein-repellent composite would help repel bacterial attachment. Previous studies reported that most proteins adsorb preferentially onto hydrophobic surfaces [53]. MPC is a methacrylate with phospholipid polar group in the side chain [27]. Phospholipids are a major component of all cell membranes as they can form lipid 
bilayers [54]. MPC polymers are highly hydrophilic [27] and well known to reduce protein adsorption and bacterial adhesion [28, 29]. Regarding the protein-repellent mechanism, it was reported that, in the hydrated MPC polymer, there is an abundance of free water but no bound water [27]. The bound water would result in protein adsorption [28, 55]. The large amount of free water around the phosphorylcholine groups could detach proteins, thereby repelling protein adsorption $[28,55]$. The present study showed that incorporating 3\% MPC into composite reduced protein adsorption by an order of magnitude, which indeed led to much less biofilm formation of the four periodontal pathogens.

Furthermore, this study showed that combining MPC with DMAHDM achieved greater inhibition of periodontal pathogens than using either agent alone. The mode of antibacterial action for QAMs is suggested to be contact-inhibition [21]. The positively-charged quaternary amine $\mathrm{N}^{+}$interacts with the negatively-charged cell membrane of bacteria, leading to membrane disruption and cytoplasmic leakage [21]. This contact-killing mechanism would indicate that, when a salivary protein pellicle separates the antibacterial resin surface from the overlaying biofilm, the antibacterial effects of the resin could be reduced [21]. However, because MPC could repel the proteins from covering the composite surface, it would lead to direct contact of the composite surface with bacteria, thus enhancing the contact-killing efficacy. This factor contributed to the reduction in biofilm CFU by an order of magnitude over that via DMAHDM alone without MPC.

The four pathogens showed different extent of being inhibited by the composite. The extent of CFU reduction via EBPM+3DMAHDM+3MPC was the greatest for $P$. gingivalis (reduced to $0.008 \%$ of the control CFU), and the least for F. nucleatum (reduced to $0.014 \%$ of 
control CFU). The killing efficacy of EBPM+3DMAHDM+3MPC composite against the four species was: $F$. nucleatum $<P$ intermedia $=$ A. actinomycetemcomitans $<P$. gingivalis. The higher sensitivity of $P$. gingivalis to DMAHDM may have significant clinical implications because it is regarded as the key pathogen of periodontitis [5]. A previous study also showed that $P$. gingivalis was more easily inhibited than $P$. intermedia, A. actinomycetemcomitans and F. nucleatum [56]. Previous studies also showed that the susceptibility of $F$. nucleatum depended on the different types of antibacterial agents. For example, F. nucleatum was more sensitive to nano-silver and nano-copper than $P$. gingivalis, $P$. intermedia and $A$. actinomycetemcomitans [56]. However, for nano-zinc, F. nucleatum was harder to kill than $P$. gingivalis [56]. In addition, the $F$. nucleatum $\mathrm{CFU}$ had a smaller reduction than $P$. gingivalis in dual-species biofilms with a povidone-iodine treatment [57]. This is consistent with the present study showing that $F$. nucleatum was less susceptible to EBPM+3DMAHDM+3MPC than $P$. gingivalis, $P$. nigrencens, $P$. intermedia and A. actinomycetemcomitans.

It should be noted that biofilm CFU values on control composites were also different for the four different species. A previous study indicated that different periodontal pathogens exhibited different deoxyribonucleases (DNase) activity [58]. The physiological roles for DNase production included the liberation of nucleotides that could be used to confer growth advantage. However, although different species grew differently and had different CFU on control composites, biofilm CFUs on EBPM+3DMAHDM+3MPC for the four periodontal pathogens were all reduced by nearly $4 \log$. Hence, EBPM+3DMAHDM+3MPC is a promising protein-repellent and antibacterial composite for Class V restorations.

EBPM+3DMAHDM+3MPC substantially reduced the metabolic activity of periodontal 
biofilms. MTT conversion is a marker of cellular metabolism, and has been used for quantifying the dehydrogenase activities of biofilms [42]. In addition, polysaccharide is synthesized by live bacteria and hence would also be related to bacterial viability. In a biofilm, bacteria are surrounded by extracellular polymeric substances (EPS) which is composed of polysaccharides, proteins and extracellular DNA [59]. EPS accounts for about $90 \%$ of the total biofilm mass [59]. In the present study, polysaccharide synthesis by all four species was substantially reduced via EBPM+3DMAHDM+3MPC. EPS protects pathogens from antibacterial agents, and contributes to the virulence and pathogenicity of pathogens via small molecule-mediated inter- and intra-species cross-talks [60]. Microbes would decelerate the synthesis of EPS when suffering from external stresses, since more energy is needed for their own survival. Therefore, the EPS reduction via EBPM+3DMAHDM+3MPC composite could reduce or even destroy the protection that the bacteria have, lessen the toxicity of the pathogens, and thus may effectively inhibit local periodontitis.

Besides inhibiting periodontal biofilms, the EBPM+3DMAHDM+3MPC composite also contained NACP with $\mathrm{Ca}$ and $\mathrm{P}$ ion release. NACP composite effectively remineralized enamel lesions and inhibited caries at composite-tooth margins, as shown previously [34, 35]. Hence, in Class V restorations, EBPM+3DMAHDM+3MPC composite with NACP could remineralize tooth root lesions and protect the surrounding root structures. Because the antibacterial monomer is covalently bonded with and immobilized in the resin, the antibacterial effect is durable and not lost over time $[43,61]$. However, further study is needed to investigate EBPM+3DMAHDM+3MPC for Class V restorations, including its long-term inhibition of periodontal and cariogenic biofilms in a clinically relevant manner. 


\section{Conclusions}

This study developed a bioactive nanocomposite for Class V restorations with a combination of protein-repellent and antibacterial capabilities to combat periodontal biofilms. The effects of MPC + DMAHDM in EBPM composite with remineralizing NACP on mechanical properties, water sorption, protein adsorption, and periodontitis-related biofilms were determined for the first time. The mechanical properties of the nanocomposite were similar to those of a commercial composite used in Class V restorations. The composite with 3\% DMAHDM showed a strong antibacterial capability. The composite with 3\% MPC was strongly protein- and bacteria-repellent. Furthermore, the use of dual agents, 3\% DMAHDM + 3\% MPC, in the composite achieved the greatest reduction in biofilm growth, metabolic activity and polysaccharide production. Biofilm CFU counts were reduced by nearly 4 orders of magnitude for all four periodontal pathogens. Therefore, the new EBPM+3DMAHDM+3MPC composite is promising for Class $\mathrm{V}$ restorations to inhibit periodontal pathogens, combat periodontitis and protect the periodontium.

\section{Acknowledgement}

We thank Dr. A. F. Fouad of University of Maryland, School of Dentistry for donation of bacteria strains. This work was supported by NIH R01 DE17974 (HX), National Science Foundation of China 81400487 (LW), 81200820 (XX), Youth Fund of Science and Technology of Jilin Province 20150520043JH (LW), China Postdoctoral Science Foundation 2015M581405 (LW), China Scholarship Council (LW), University of Maryland School of 
Dentistry bridging fund (HX) and University of Maryland seed grant (HX).

\section{Reference}

1. E.R. Kleinman, P.R. Harper, J. E. Gallagher, Trends in nhs primary dental care for older people in england: Implications for the future, Gerodontology 26 (2009) 193-201.

2. K.M. Heegaard, P. Holm Pedersen, A. Bardow, U.A. Hvidtfeldt, M. Grønbæk, K. Avlund, The copenhagen oral health senior cohort: Design, population and dental health, Gerodontology 28 (2011) 165-176.

3. S. Fure, Ten-year incidence of tooth loss and dental caries in elderly swedish individuals, Caries Res. 37 (2003) 462-469.

4. N. Ravald, C.S. Johansson, Tooth loss in periodontally treated patients. A long-term study of periodontal disease and root caries, J. Clin. Periodontol. 39 (2012) 73-79.

5. N. Beyth, A.J. Domb, E.I. Weiss, An in vitro quantitative antibacterial analysis of amalgam and composite resins, J. Dent. 35 (2007) 201-206.

6. P.S. Kumar, A.L. Griffen, M.L. Moeschberger, E.J. Leys, Identification of candidate periodontal pathogens and beneficial species by quantitative 16 s clonal analysis, J. Clin. Microbiol. 43 (2005) 3944-3955.

7. G. Hajishengallis, R.P. Darveau, M.A. Curtis, The keystone-pathogen hypothesis, Nat. Rev. Microbiol. 10 (2012) 717-725.

8. D. Fteita, E. Könönen, E. Söderling, U.K. Gürsoy, Effect of estradiol on planktonic growth, coaggregation, and biofilm formation of the prevotella intermedia group bacteria, Anaerobe 27 (2014) 7-13. 
9. D.H. Fine, K. Markowitz, D. Furgang, K. Fairlie, J. Ferrandiz, C. Nasri, et al., Aggregatibacter actinomycetemcomitans and its relationship to initiation of localized aggressive periodontitis: Longitudinal cohort study of initially healthy adolescents, J. Clin. Microbiol. 45 (2007) 3859-3869.

10. P. E. Kolenbrander, J. London, Adhere today, here tomorrow: Oral bacterial adherence, J. Bacteriol. 175 (1993) 3247.

11. P. Diaz, P. Zilm, A. Rogers, Fusobacterium nucleatum supports the growth of porphyromonas gingivalis in oxygenated and carbon-dioxide-depleted environments, Microbiology 148 (2002) 467-472.

12. J.L. Ferracane, Resin composite-state of the art, Dent. Mater. 27 (2011) 29-38.

13. J.L. Drummond, Degradation, fatigue, and failure of resin dental composite materials, $J$. Dent. Res. 87 (2008) 710-719.

14. B.S. Lim, J. Ferracane, R. Sakaguchi, J. Condon, Reduction of polymerization contraction stress for dental composites by two-step light-activation, Dent. Mater. 18 (2002) 436-444.

15. D.C. Watts, A. Marouf, A. Al-Hindi, Photo-polymerization shrinkage-stress kinetics in resin-composites: Methods development, Dent. Mater. 19 (2003) 1-11.

16. Y.J. Wei, N. Silikas, Z.T. Zhang, D.C. Watts, Hygroscopic dimensional changes of self-adhering and new resin-matrix composites during water sorption/desorption cycles, Dent. Mater. 27 (2011) 259-266.

17. S. Gong, L. Niu, L.K. Kemp, C.K. Yiu, H. Ryou, Y. Qi, et al., Quaternary ammonium silane-functionalized, methacrylate resin composition with antimicrobial activities and 
self-repair potential, Acta Biomater. 8 (2012) 3270-3282.

18. X. Xu, Y. Wang, S. Liao, Z.T. Wen, Y. Fan, Synthesis and characterization of antibacterial dental monomers and composites, J. Biomed. Mater. Res. B 100 (2012) $1151-1162$.

19. F. Li, J. Chen, Z. Chai, L. Zhang, Y. Xiao, M. Fang, et al., Effects of a dental adhesive incorporating antibacterial monomer on the growth, adherence and membrane integrity of streptococcus mutans, J. Dent. 37 (2009) 289-296.

20. S. Imazato, Antibacterial properties of resin composites and dentin bonding systems, Dent. Mater. 19 (2003) 449-457.

21. N. Beyth, I. Yudovin-Farber, R. Bahir, A.J. Domb, E. I. Weiss, Antibacterial activity of dental composites containing quaternary ammonium polyethylenimine nanoparticles against streptococcus mutans, Biomaterials 27 (2006) 3995-4002.

22. S. Imazato, Bio-active restorative materials with antibacterial effects: New dimension of innovation in restorative dentistry, Dent. Mater. J. 28 (2009) 11-19.

23. C. Zhou, M.D. Weir, K. Zhang, D. Deng, L. Cheng, H.H. Xu, Synthesis of new antibacterial quaternary ammonium monomer for incorporation into cap nanocomposite, Dent. Mater. 29 (2013) 859-870.

24. F. Li, M.D. Weir, H.H. Xu, Effects of quaternary ammonium chain length on antibacterial bonding agents, J. Dent. Res. 92 (2013) 932-938.

25. N. Namba, Y. Yoshida, N. Nagaoka, S. Takashima, K. Matsuura-Yoshimoto, H. Maeda, et al., Antibacterial effect of bactericide immobilized in resin matrix, Dent. Mater. 25 (2009) 424-430. 
26. R. Müller, A. Eidt, K.A. Hiller, V. Katzur, M. Subat, H. Schweik1, et al., Influences of protein films on antibacterial or bacteria-repellent surface coatings in a model system using silicon wafers, Biomaterials 30 (2009) 4921-4929.

27. K. Ishihara, T. Ueda, N. Nakabayashi, Preparation of phospholipid polymers and their properties as polymer hydrogel membranes, Polym. J. 22 (1990) 355-360.

28. K. Ishihara, H. Nomura, T. Mihara, K. Kurita, Y. Iwasaki, N. Nakabayashi, Why do phospholipid polymers reduce protein adsorption?, J. Biomed. Mater. Res. 39 (1998) 323-330.

29. J. Sibarani, M. Takai, K. Ishihara, Surface modification on microfluidic devices with 2-methacryloyloxyethyl phosphorylcholine polymers for reducing unfavorable protein adsorption, Colloid. Surface. B 54 (2007) 88-93.

30. N. Zhang, C. Chen, M.D. Weir, Y. Bai, H.H. Xu, Antibacterial and protein-repellent orthodontic cement to combat biofilms and white spot lesions, J. Dent. 43 (2015) 1529-1538. 31. N. Zhang, K. Zhang, M.A.S. Melo, C. Chen, A. F. Fouad, Y. Bai, et al., Novel protein-repellent and biofilm-repellent orthodontic cement containing 2-methacryloyloxyethyl phosphorylcholine, J. Biomed. Mater. Res. Part B (2015) DOI: $10.1002 / j b m \cdot b .33444$.

32. L. Zhang, M.D. Weir, L.C. Chow, J.M. Antonucci, J. Chen, H.H. Xu, Novel rechargeable calcium phosphate dental nanocomposite, Dent. Mater. 32 (2016) 285-293.

33. J.M. Antonucci, D.N. Zeiger, K. Tang, S. Lin-Gibson, B.O. Fowler, N.J. Lin, Synthesis and characterization of dimethacrylates containing quaternary ammonium functionalities for dental applications, Dent. Mater. 28 (2012) 219-228. 
34. M.D. Weir, L.C. Chow, H.H. Xu, Remineralization of demineralized enamel via calcium phosphate nanocomposite, J. Dent. Res. 91 (2012) 979-984.

35. H.H. Xu, J.L. Moreau, L. Sun, L.C. Chow, Nanocomposite containing amorphous calcium phosphate nanoparticles for caries inhibition, Dent. Mater. 27 (2011) 762-769.

36. M. Trujillo-Lemon, J. Ge, H. Lu, J. Tanaka, J.W. Stansbury, Dimethacrylate derivatives of dimer acid, J. Polym. Sci. Pol. Che. 44 (2006) 3921-3929.

37. ISO. Standard, 4049, 2009, Dentistry-polymer-based restorative materials, International Organization for Standardization, Geneva, Switzerland (2009).

38. K. Ishihara, N.P. Ziats, B.P. Tierney, N. Nakabayashi, J.M. Anderson, Protein adsorption from human plasma is reduced on phospholipid polymers, J. Biomed. Mater. Res. 25 (1991) $1397-1407$.

39. S. Imazato, A. Ehara, M. Torii, S. Ebisu, Antibacterial activity of dentine primer containing mdpb after curing, J. Dent. 26 (1998) 267-271.

40. N. Ebi, S. Imazato, Y. Noiri, S. Ebisu, Inhibitory effects of resin composite containing bactericide-immobilized filler on plaque accumulation, Dent. Mater. 17 (2001) 485-491.

41. Y. Saito, R. Fujii, K.I. Nakagawa, H. Kuramitsu, K. Okuda, K. Ishihara, Stimulation of fusobacterium nucleatum biofilm formation by porphyromonas gingivalis, Oral Microbiol. Immunol. 23 (2008) 1-6.

42. C. Chen, M.D. Weir, L. Cheng, N.J. Lin, S. Lin-Gibson, L.C. Chow, et al., Antibacterial activity and ion release of bonding agent containing amorphous calcium phosphate nanoparticles, Dent. Mater. 30 (2014) 891-901.

43. L. Cheng, M. Weir, K. Zhang, S. Xu, Q. Chen, X. Zhou, et al., Antibacterial 
nanocomposite with calcium phosphate and quaternary ammonium, J. Dent. Res. 91 (2012) 460-466.

44. N. Zhang, M.D. Weir, E. Romberg, Y. Bai, H.H. Xu, Development of novel dental adhesive with double benefits of protein-repellent and antibacterial capabilities, Dent. Mater. 31 (2015) 845-854.

45. F. Stahl, S.H. Ashworth, K.D. Jandt, R.W. Mills, Light-emitting diode (led) polymerisation of dental composites: Flexural properties and polymerisation potential, Biomaterials 21 (2000) 1379-1385.

46. J.L. Ferracane, Hygroscopic and hydrolytic effects in dental polymer networks, Dent. Mater. 22 (2006) 211-222.

47. D.F.G. Cefaly, E.B. Franco, R.F.L. Mondelli, P.A.S. Francisconi, M.F.d.L. Navarro, Diametral tensile strength and water sorption of glass-ionomer cements used in atraumatic restorative treatment, J. Appl. Oral Sci. 11 (2003) 96-101.

48. P.I. Eke, B.A. Dye, L. Wei, G.D. Slade, G.O. Thornton-Evans, W.S. Borgnakke, et al., Update on prevalence of periodontitis in adults in the united states: Nhanes 2009 to 2012, J. Periodontol. 86 (2015) 611-622.

49. J.K. Kim, L.A. Baker, H. Seirawan, E.M. Crimmins, Prevalence of oral health problems in us adults, nhanes 1999-2004: Exploring differences by age, education, and race/ethnicity, Spec. Care Dent. 32 (2012) 234-241.

50. A. Ashimoto, C. Chen, I. Bakker, J. Slots, Polymerase chain reaction detection of 8 putative periodontal pathogens in subgingival plaque of gingivitis and advanced periodontitis lesions, Oral Microbiol. Immunol. 11 (1996) 266-273. 
51. B.L. Pihlstrom, B.S. Michalowicz, N.W. Johnson, Periodontal diseases, Lancet 366 (2005) 1809-1820.

52. R.M. Donlan, J.W. Costerton, Biofilms: Survival mechanisms of clinically relevant microorganisms, Clin. Microbiol. Rev. 15 (2002) 167-193.

53. M. Katsikogianni, Y. Missirlis, Concise review of mechanisms of bacterial adhesion to biomaterials and of techniques used in estimating bacteria-material interactions, Eur. Cells Mater. 8 (2004)

54. S. Mashaghi, T. Jadidi, G. Koenderink, A. Mashaghi, Lipid nanotechnology, Int. J. Mol. Sci. 14 (2013) 4242-4282.

55. T. Goda, T. Konno, M. Takai, K. Ishihara, Photoinduced phospholipid polymer grafting on parylene film: Advanced lubrication and antibiofouling properties, Colloid. Surface. B 54 (2007) 67-73.

56. M.A. Vargas-Reus, K. Memarzadeh, J. Huang, G.G. Ren, R.P. Allaker, Antimicrobial activity of nanoparticulate metal oxides against peri-implantitis pathogens, Int. J. Antimicrob. Ag. 40 (2012) 135-139.

57. Y. Hosaka, A. Saito, R. Maeda, C. Fukaya, S. Morikawa, A. Makino, et al., Antibacterial activity of povidone-iodine against an artificial biofilm of porphyromonas gingivalis and fusobacterium nucleatum, Arch. Oral Biol. 57 (2012) 364-368.

58. L. Palmer, I. Chapple, H. Wright, A. Roberts, P. Cooper, Extracellular deoxyribonuclease production by periodontal bacteria, J. Periodontal Res. 47 (2012) 439-445.

59. D. McDougald, S.A. Rice, N. Barraud, P.D. Steinberg, S. Kjelleberg, Should we stay or should we go: Mechanisms and ecological consequences for biofilm dispersal, Nat. Rev. 
Microbiol. 10 (2012) 39-50.

60. M.T. Pöllänen, A. Paino, R. Ihalin, Environmental stimuli shape biofilm formation and the virulence of periodontal pathogens, Int. J. Mol. Sci. 14 (2013) 17221-17237.

61. K. Zhang, L. Cheng, E.J. Wu, M.D. Weir, Y.X. Bai, H.H. Xu, Effect of water-aging on dentin bond strength and anti-biofilm activity of bonding agent containing antibacterial monomer dimethylaminododecyl methacrylate, J. Dent. 41 (2013) 504-513.

\section{Figure captions}

[1]. Mechanical property of composites: (A) Flexural strength and (B) elastic modulus (mean $\pm \mathrm{SD} ; n=6$ ), and (C) water sorption (mean $\pm \mathrm{SD} ; n=5$ ). In each plot, bars with dissimilar letters are significantly different from each other $(p<0.05)$.

[2]. Protein adsorption onto composite surfaces (mean $\pm \mathrm{SD} ; n=6$ ). The composite with $3 \%$ DMAHDM $+3 \%$ MPC had much less protein adsorption, which was about $1 / 9$ that of commercial control composite $(p<0.05)$. Bars with dissimilar letters are significantly different from each other $(p<0.05)$.

[3]. Representative live/dead staining images of 2-day biofilms of four species of periodontal pathogens on the five composites: (A-D) Commercial composite control, (E-H) EBPM composite control, (I-L) EBPM+3MPC, (M-P) EBPM+3DMAHDM, and (Q-T) EBPM+3DMAHDM+3MPC. All images had the same scale bar as shown in (A). Live bacteria were stained green. Dead bacteria were stained red. Composites without DMAHDM had primarily live bacteria. EBPM+3DMAHDM+3MPC had much less bacterial adhesion, and the biofilms consisted of primarily dead bacteria. 
[4]. CFU counts of 2-day biofilms on composites: (A) P. gingivalis, (B) P. intermedia, (C) A. actinomycetemcomitans, and (D) F. nucleatum (mean \pm SD; $n=6$ ). Note the log scale for the y-axis. Bars with dissimilar letters are significantly different from each other $(p<$ $0.05)$

[5]. Metabolic activity of 2-day biofilms grown on composites measured by the MTT assay: (A) P. gingivalis, (B) P. intermedia, (C) A. actinomycetemcomitans, and (D) F. nucleatum (mean $\pm \mathrm{SD} ; n=6$ ). Dissimilar letters indicate significantly different values $(p<0.05$ ).

[6]. Polysaccharide synthesis by bacteria in 2-day biofilms grown on composites: (A) $P$. gingivalis, (B) P. intermedia, (C) A. actinomycetemcomitans, and (D) F. nucleatum (mean $\pm \mathrm{SD} ; n=6)$. Bars with dissimilar letters indicate significantly different values ( $p$ $<0.05)$. 


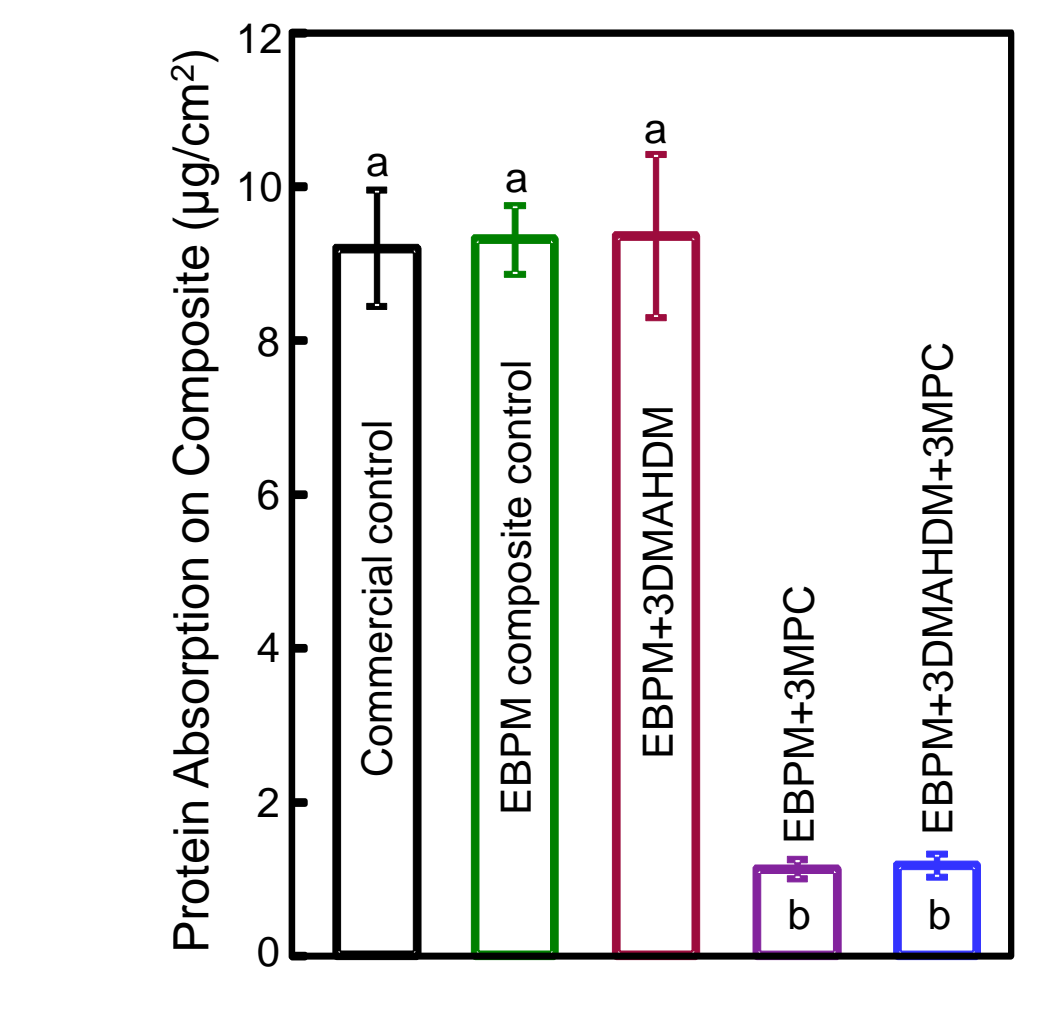

Fig. 2

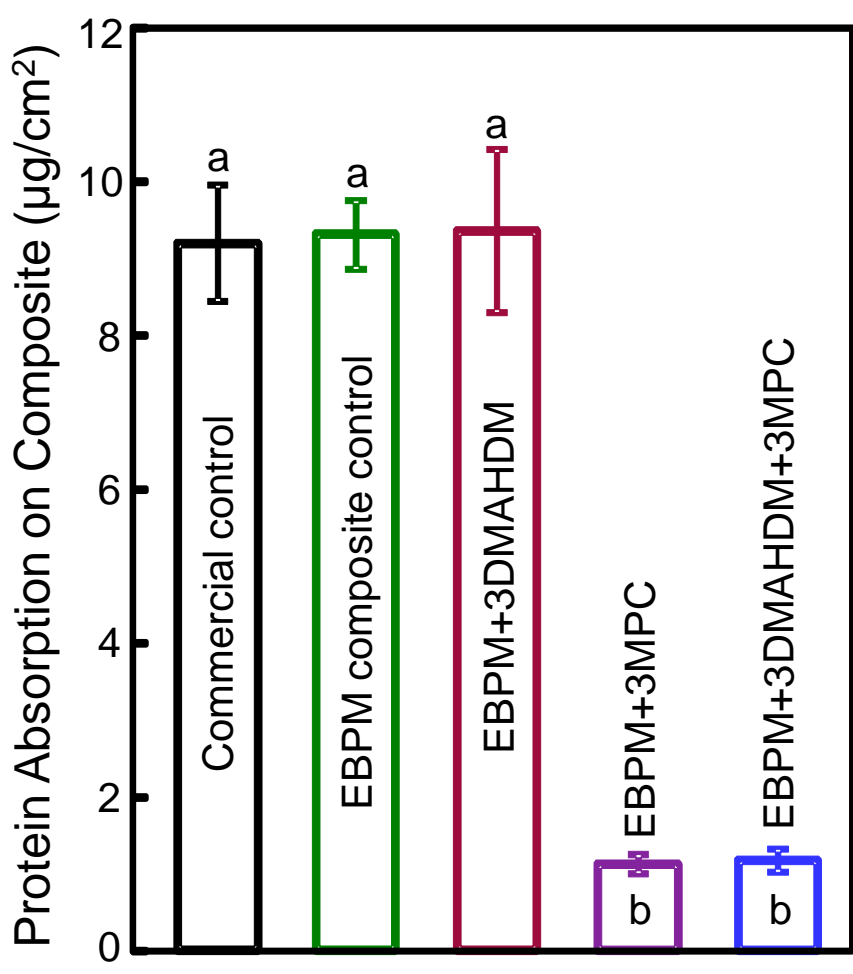

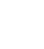

\section{Figure2}



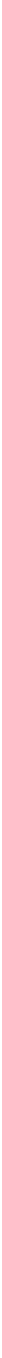

(K)

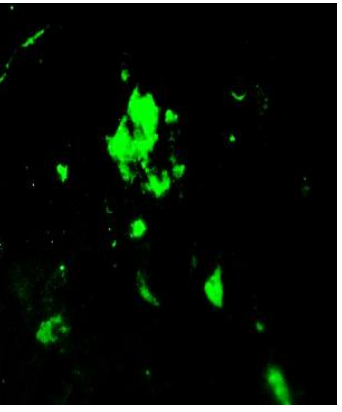

(L)

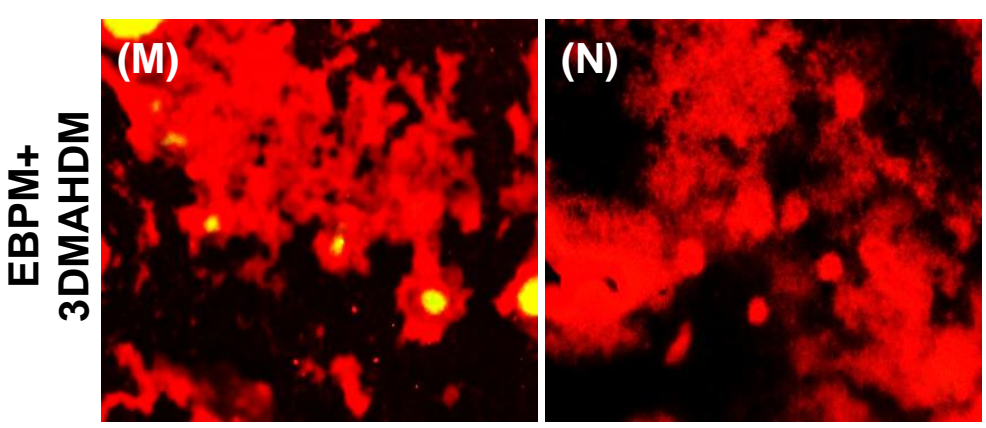

(O)

(P)

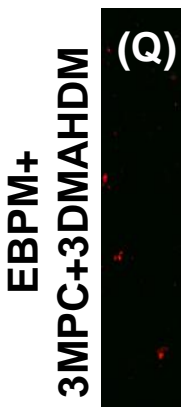

(R)

(S)

(T)

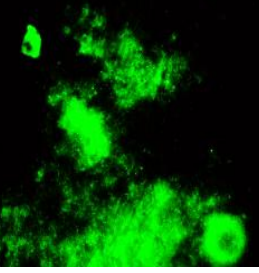

Fig. 3 

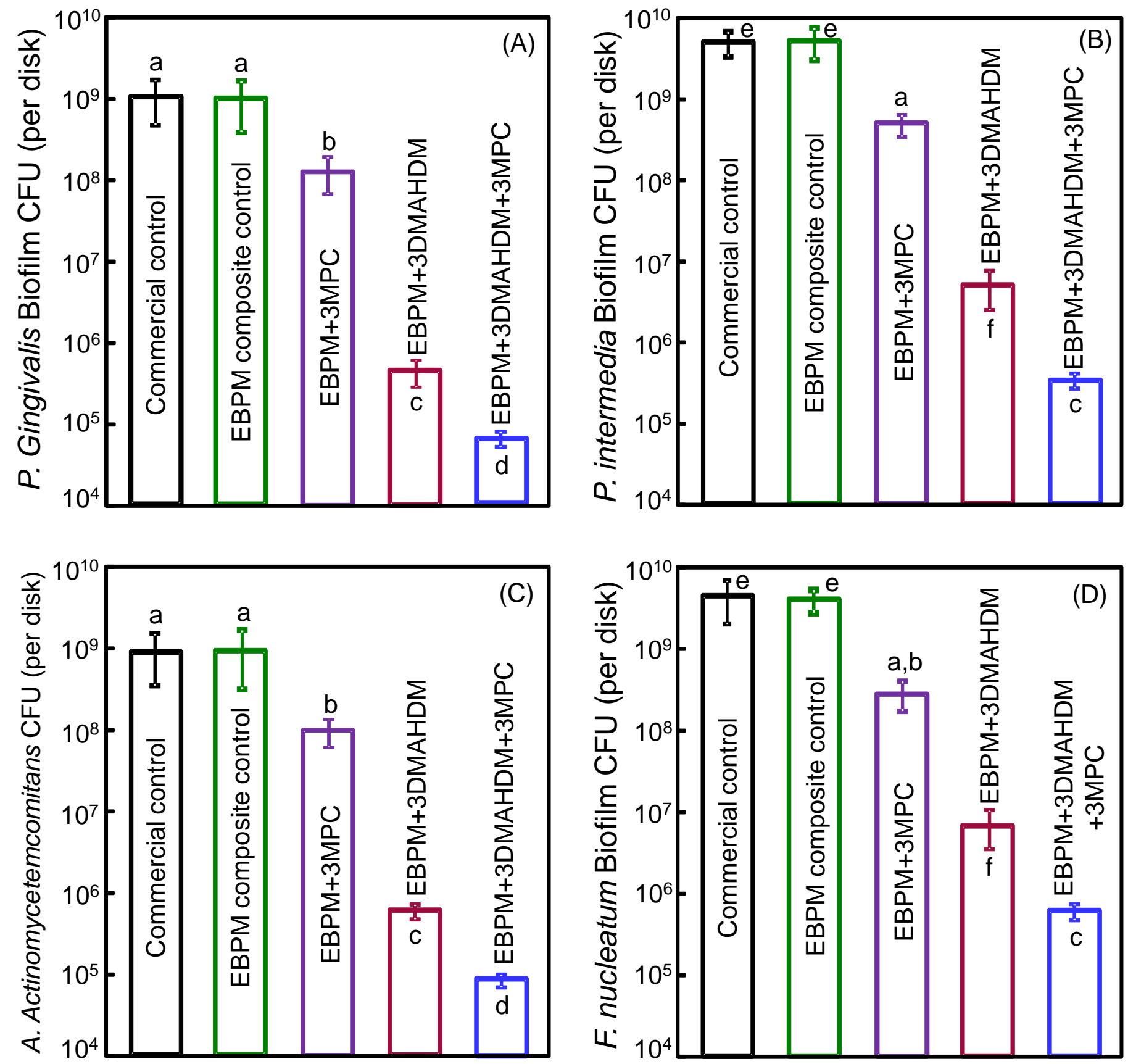

Fig. 4 

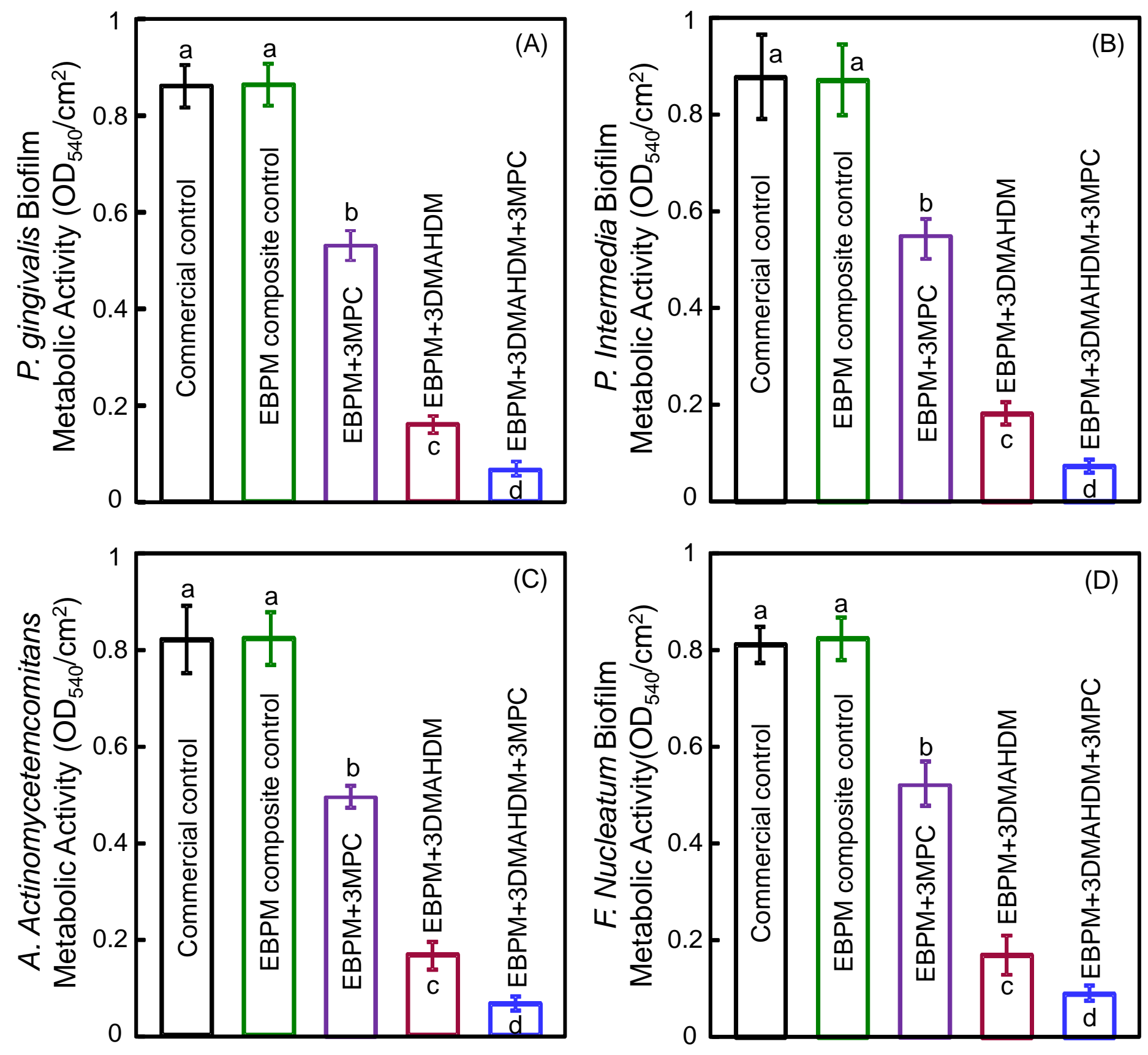

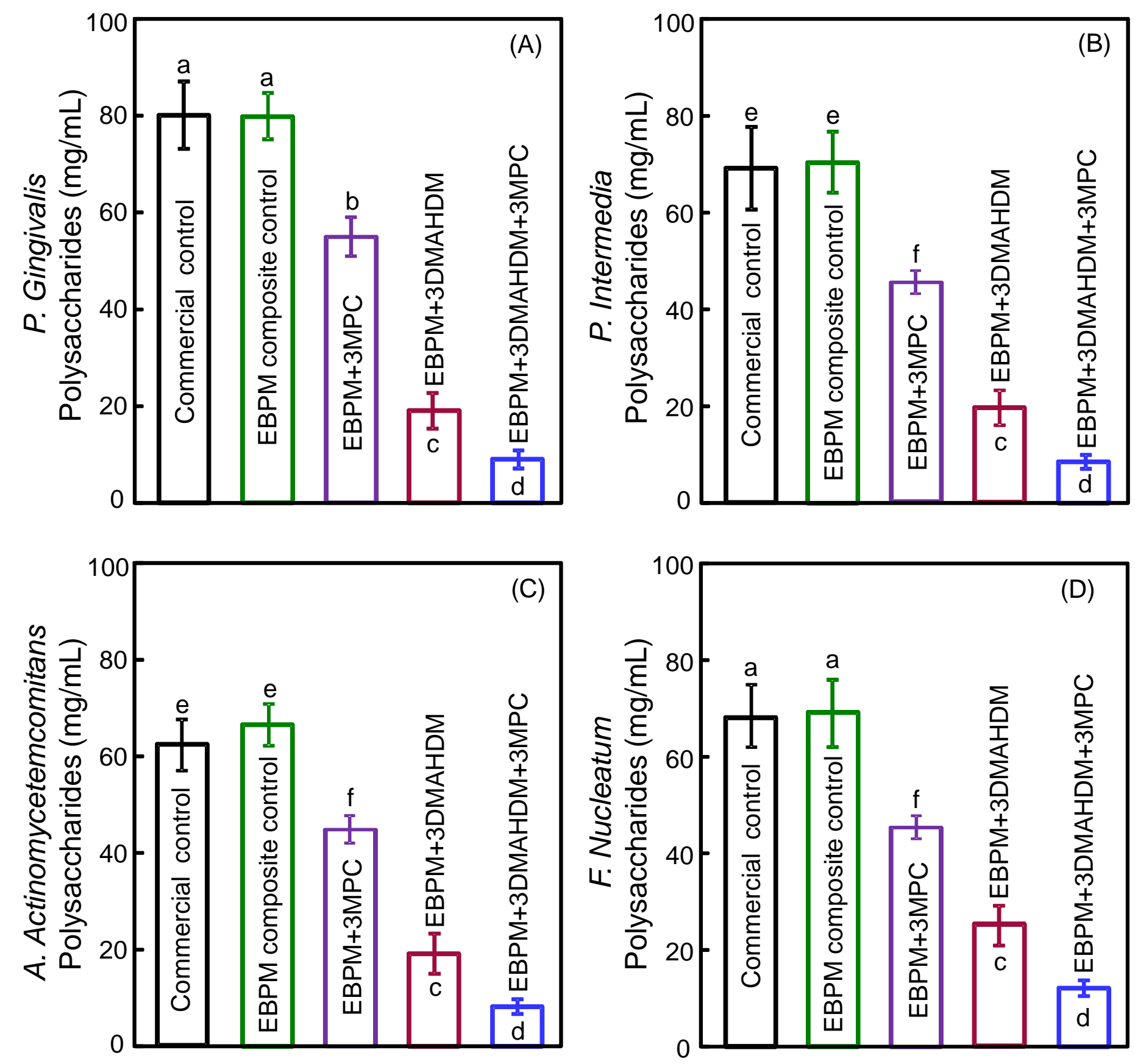